\title{
Indicação Geográfica: mapeamento e análise sistêmica das publicações
}

\section{Geographical Indication: mapping and systemic analysis of publications}

\author{
Adriana Araújo Maués ${ }^{1}$ \\ Fernando Richartz ${ }^{1}$ \\ ${ }^{1}$ Universidade Federal de Santa Catarina, Florianópolis, SC, Brasil
}

\begin{abstract}
Resumo
Este artigo tem como objetivo realizar um mapeamento das pesquisas científicas sobre Indicação Geográfica (IG) de forma a identificar seu panorama atual. Observou-se que a IG é um tema que tem ganhado visibilidade no meio científico com publicações relevantes de outros autores da Revista de Prospecção. Por meio de busca em bases de dados, foi possível obter a seleção de um Portfólio Bibliográfico (PB) relevante de 36 artigos, de acordo com os critérios definidos na pesquisa. Destes, tem-se o artigo de Sarah K. Bowen (2010) como destaque do portfólio. O principal periódico é o World Development com sete artigos do PB publicados. Por meio da relação entre os principais artigos da análise bibliométrica com as características da análise sistêmica, conclui-se que um artigo de destaque em Indicação Geográfica possui abordagem qualitativa, com a utilização de, principalmente, entrevistas, sendo possível identificar diversos fatores considerados como desafios e vantagens na obtenção da Indicação Geográfica.
\end{abstract}

Palavras-chave: Indicação Geográfica. Análise Bibliométrica. Mapeamento de Artigos.

\begin{abstract}
This article aims to map scientific research on geographical indication in order to identify the current panorama. IG is a topic that has gained visibility in the scientific environment with relevant publications by other authors of the Prospecting Journal. By searching databases, we obtained the selection of a relevant bibliographic portfolio (BP) of 36 articles, according to the criteria defined in the research. The most relevant is the article of Sarah K. Bowen (2010). The main journal is the World Development with 7 published articles of the BP. Finally, between the main articles of the bibliometric analysis and the characteristics of the systemic analysis, it is concluded that a prominent article in geographical indication has a qualitative approach, with the main use of interviews, being possible to identify several factors considered as challenges and advantages in obtaining the geographical indication.
\end{abstract}

Keywords: Geographical Indication. Bibliometric Analysis. Article Mapping.

Área Tecnológica: Propriedade Intelectual. Indicações Geográficas. 


\section{Introdução}

A Indicação Geográfica (IG) é um direito de Propriedade Intelectual autônomo, reconhecido nacional e internacionalmente e de uso coletivo pelos produtores ou prestadores de serviço estabelecidos no local, desde que cumpridas determinadas condições. O registro de IG é conferido a produtos ou serviços que possuem qualidade ou reputação associadas à sua origem geográfica (MAPA, 2019). Segundo a legislação brasileira, podem ser de dois tipos: Indicação de Procedência (IP) ou Denominação de Origem (DO) (INPI, 2018).

Observou-se que a Indicação Geográfica é um tema que tem ganhado visibilidade no meio científico com publicações relevantes explorando os diversos aspectos referentes à proteção $e$ suas consequências, conforme demonstram vários trabalhos, como os de Marins e Cabral (2015) e Flores, Tonietto e Taffarel (2019).

Nesse sentido, identificar o estado da arte na temática da Indicação Geográfica pode contribuir para estruturar os resultados obtidos em diversas pesquisas sobre o tema, auxiliando no desenvolvimento científico de estudos futuros. Assim, a pergunta de pesquisa a ser respondida é: qual o atual panorama das pesquisas com relação ao tema Indicação Geográfica de acordo com procedimentos estruturados para a construção do conhecimento? Esta pesquisa tem como objetivo geral realizar um mapeamento de artigos científicos a respeito das Indicações Geográficas, a fim de identificar o panorama atual e a evolução do tema. Para tanto, foram definidos os seguintes objetivos específicos:

a) Efetuar pesquisa em bases de dados para seleção de um portfólio bibliográfico (PB) relevante;

b) Realizar análise bibliométrica dos artigos do PB e das suas referências;

c) Efetuar análise sistêmica (análise de conteúdo) com os artigos do PB a respeito das Indicações Geográficas; e

d) Identificar lacunas e oportunidades de pesquisa com base na análise sistêmica.

Este artigo é original, pois se dispõe a consolidar os resultados das pesquisas acerca das Indicações Geográficas por meio do mapeamento de artigos. Além disso, o estudo é viável, uma vez que utiliza como fonte de coleta de informações trabalhos disponíveis em bases de dados de acesso público.

\section{Revisão da Literatura}

Os Acordos Internacionais (Convenção de Paris, Acordo de Madrid, Acordo de Lisboa) estabeleceram uma base conceitual para as Indicações Geográficas (ILBERT; PETIT, 2009), porém o reconhecimento universal das IGs veio com a incorporação dos resultados da Rodada do Uruguai de Negociações Comerciais Multilaterais do GATT, assinada em Marraquexe em 12 de abril de 1994, com a instituição do Acordo sobre Aspectos Relativos aos Direitos de Propriedade Intelectual Concernentes ao Comércio (ADPIC), ou Agreement on Trade-Related Aspects of Property Rights (TRIPs). 
Na União Europeia, o Regulamento (UE) n. 1.151/2012 estabelece as definições de Denominações de Origem e Indicações Geográficas. No Brasil, a Lei n. 9.279/1996 aborda a questão da Indicação Geográfica no Título IV, do artigo 176 ao artigo 182.

Art. 177 Considera-se Indicação de Procedência o nome geográfico de país, cidade, região ou localidade de seu território, que se tenha tornado conhecido como centro de extração, produção ou fabricação de determinado produto ou de prestação de determinado serviço.

[...]

Art. 178 Considera-se Denominação de Origem o nome geográfico de país, cidade, região ou localidade de seu território, que designe produto ou serviço cujas qualidades ou características se devam exclusiva ou essencialmente ao meio geográfico, incluídos fatores naturais e humanos. (BRASIL, 2019, grifos nossos)

No Brasil, seu registro é competência do Instituto Nacional da Propriedade Industrial (INPI), autarquia federal criada em 1970 e vinculada ao Ministério da Economia, conforme o prevê o Decreto n. 9.660/2019. As condições para o registro são estabelecidas pela Instrução Normativa n. 095/2018 que trouxe importantes novidades, por exemplo, a alteração e a supressão de nome do produto/serviço, a alteração da representação gráfica/figurativa da IG, bem como a alteração do nome do "Regulamento de Uso" para "Caderno de Especificações Técnicas".

Os legitimados para requerer o registro são substitutos processuais representativos da coletividade localizada no território definido pelo IG, isto é, a associação, o sindicato ou outra entidade representativa da cadeia produtiva do respectivo produto/serviço.

Ademais, a proteção concedida por meio da Indicação Geográfica é declaratória, na medida em que pressupõe condições preexistentes, quais sejam: a reputação ou a influência do meio geográfico. O pedido deverá se referir a apenas um nome geográfico, e os documentos exigidos devem estar em conformidade com a modalidade pretendida, IP (produtos ou serviços reconhecidos pela produção, fabricação ou extração de uma determinada localidade) ou DO (características do produto ou serviço devido ao meio geográfico, incluindo fatores naturais e humanos) (MAPA, 2019).

\section{Metodologia}

O enquadramento metodológico utilizado nesta pesquisa é apresentado a seguir com o detalhamento dos procedimentos para a coleta e análise dos dados. Nesse sentido, o tópico foi dividido em processo de busca de artigos e instrumento de intervenção.

\subsection{Processo de Busca de Artigos}

A pesquisa se classifica como qualitativa, pois busca um entendimento mais detalhado dos artigos analisados para gerar conhecimento a respeito do tema das Indicações Geográficas, contribuindo, assim, para a evolução da pesquisa científica (RICHARDSON, 1999). 
O procedimento técnico utilizado foi a pesquisa bibliográfica por meio de uma seleção de artigos disponíveis em bases de dados (Scopus, Web of Science, EBSCO), constituindo o portfólio bibliográfico.

Para essa primeira etapa de seleção de artigos, foram definidas as palavras-chave para o eixo de pesquisa referente às Indicações Geográficas. Com o intuito de ampliar o campo de investigação, utilizou-se, além do português, o inglês como idioma de busca de palavras-chave. Nesse sentido, foram utilizadas estas palavras: Indicação Geográfica; Indicação de Procedência, Denominação de Origem e Geographical Indication; Protected Designation of Origin; Protected Geographical Indication; Traditional Specialities Guaranteed.

Na sequência, foram definidas as bases de dados para consulta de artigos com a condição de permitirem o uso de expressão booleana AND, OR e permitir pesquisa em título, resumo e palavras-chave. As bases também devem estar disponíveis no portal de periódicos da CAPES. Por essa razão, foram selecionadas estas bases: Web of Science, Scopus e EBSCO.

Utilizou-se o software EndNote para gerenciar as referências coletadas. A ferramenta importa as referências de bancos de dados abertos de artigos e as organiza em grupos, formatando o conteúdo segundo as normas da ABNT.

A pesquisa ocorreu no mês de fevereiro de 2019, por isso, optou-se por restringir a busca por artigos publicados nos últimos 10 anos, isto é, de 2009 a 2019.

$\mathrm{Na}$ base de dados Web of Science refinou-se a seleção pelas áreas: law, economics, agriculture economic policy, development study, environmental study, management e business e foram encontrados 99 artigos. Repetindo a busca com as palavras-chaves em português, não houve resultado.

Prosseguindo com as buscas na base Scopus, aplicando o mesmo requisito do limite temporal de 2009-2019, apenas artigos de acesso aberto e nas áreas de environmental science, economics, econometrics and finance, agricultural and biological sciences, chegou-se a 157 resultados. Para a mesma pesquisa em português, somou-se mais dois resultados.

A pesquisa na base EBSCO, em artigos publicados entre 2009-2019 nas áreas de intelectual property, economic competition, international trade, obteve 10 resultados alinhados. Repetindo a busca com as palavras-chave em português, chegou-se a mais 24 artigos.

No total, foram encontrados 292 artigos. Sendo a base de dados Scopus aquela na qual foram encontrados o maior número de artigos, seguida pela Web of Science e EBSCO. Do resultado da busca, dois artigos foram selecionados de forma aleatória para o teste de aderência de palavras-chave. Neste estudo não foram encontradas novas palavras relevantes para o propósito da pesquisa, assim os 292 resultados constituem o Banco de Artigos Brutos.

\subsection{Instrumento de Intervenção}

O instrumento de intervenção utilizado para a realização desta pesquisa foi o Knowledge Development Process - Constructivist (ProKnow-C). Essa metodologia de filtragem de artigos foi criada em 2009 na Universidade Federal de Santa Catarina pelo Laboratório de Metodologias Multicritério em apoio à Decisão (LabMCDA) (ENSSLIN; ENSSLIN; PINTO, 2013; TASCA et al., 2010) e se consolidou em 2010 a partir de publicações em seu formato mais recente. Essa 
ferramenta permite, por meio de uma sequência estruturada de procedimentos a serem seguidos, a seleção de artigos científicos, constituindo o Portfólio Bibliográfico (PB).

O processo define desde os mecanismos de busca até a filtragem e a seleção do portfólio dos artigos mais relevantes a respeito de determinado tema (AFONSO et al., 2011).

O ProKnow-C utiliza-se de quatro etapas principais: i) seleção de um portfólio de artigos sobre o tema de pesquisa (PB); ii) análise bibliométrica dos artigos presentes no $\mathrm{PB}$; iii) análise sistêmica (ou de conteúdo) do PB; e iv) definição de oportunidades de pesquisa com base nas lacunas observadas na análise sistêmica (CHAVES et al., 2013). Ao final do processo, o pesquisador conhecerá o estado da arte acerca do tema analisado.

Na seleção do portfólio bibliográfico ocorre a

[...] definição dos eixos de pesquisa; definição das palavras-chave; seleção das bases de dados; busca de artigos nas bases de dados; filtragem dos artigos (envolve leitura, relevância científica e disponibilidade); teste de aderência das palavras-chave e teste de representatividade de portfólio bibliográfico. (RICHARTZ, 2013, p. 3)

Na etapa bibliométrica, o pesquisador, por meio de critérios à sua escolha, irá analisar os artigos de maior relevância acerca do tema, seus autores mais consagrados, as bases de dados com maior número de artigos científicos sobre o tema, o número de citações, entre outros.

De acordo com os critérios definidos na pesquisa, foi realizada a análise sistêmica, interpretando-se os artigos do PB. Para a escolha dos critérios, levou-se em consideração o tema das Indicações Geográficas e as vantagens e desafios encontrados em sua aplicação. Assim, foram estipulados seis critérios (Quadro 1) que embasam a análise sistêmica deste estudo.

Quadro 1 - Critérios para análise sistêmica

\begin{tabular}{|c|c|}
\hline CRITÉRIOS DA BUSCA & CARACTERIZAÇÃo E ABRANGÊNCIA \\
\hline Natureza & $\begin{array}{c}\text { As pesquisas podem ser teóricas ou teórico-empíricas. } \\
\text { Qual a forma dos artigos que compõem o PB? }\end{array}$ \\
\hline Objeto & $\begin{array}{l}\text { Onde foram aplicadas as pesquisas? Verificar em } \\
\text { que localidades os estudos ocorrem. }\end{array}$ \\
\hline Técnicas & $\begin{array}{l}\text { A análise das variações pode ser realizada por meio de análise } \\
\text { estatística (quantitativa) ou por meio de discussão de pressupostos } \\
\text { teóricos (qualitativa). Quais as principais formas e técnicas de análise } \\
\text { encontradas no PB? Estudo de caso, entrevistas, documentos. }\end{array}$ \\
\hline Vantagens & $\begin{array}{l}\text { Identificar as vantagens apontadas pelos autores } \\
\text { referentes à utilização da "Indicação Geográfica". }\end{array}$ \\
\hline Desafios & $\begin{array}{c}\text { Identificar os desafios apontados pelos autores referentes } \\
\text { à utilização da "Indicação Geográfica" }\end{array}$ \\
\hline Aplicação & A pesquisa trouxe resultados práticos? Foi aplicada de fato? \\
\hline
\end{tabular}

Fonte: Elaborado pelos autores deste artigo (2019) 
Outras pesquisas utilizando o ProKnow-C como instrumento de intervenção por meio das quais este artigo está baseado são: Afonso et al. (2011); Azevedo et al. (2011); Bortoluzzi et al. (2011); Chaves et al. (2013); Ensslin et al. (2010); Ensslin, Ensslin e Pacheco (2012); Lacerda, Ensslin e Ensslin (2011); Lacerda, Ensslin e Ensslin (2012); Richartz (2013); Rosa, Ensslin e Ensslin (2009); Tasca et al. (2010), Valmorbida et al. (2011); Viana, Ensslin e Giffhorn (2011).

\section{Resultados e Discussão}

Esta seção se divide em três partes: na primeira é apresentada a filtragem do banco de artigos brutos utilizados na pesquisa; a seguir, é realizada a análise bibliométrica, segundo o nível de relevância dos autores e dos periódicos encontrados; e, por fim, é realizada a análise sistêmica por meio de critérios predefinidos.

\subsection{Filtragem do Banco de Artigos Brutos}

Verificou-se o número total de 292 artigos constantes no EndNot. Destes, após eliminação dos repetidos, restaram 281. Procedeu-se, então, à leitura dos títulos para verificar o alinhamento com o tema da Indicação Geográfica, bem como revisar novamente à procura de títulos repetidos que pudessem ter passado despercebidos na primeira checagem. Após essa etapa, sobraram 137 artigos.

Procedeu-se à análise de reconhecimento científico desses artigos, realizando a pesquisa no Google Scholar para auferir o número de citações de cada um deles e, a seguir, elencando-os em ordem decrescente de número de citações. Estipulou-se manter todos os artigos a partir de 2015, ainda que não tivessem citações pelo fato de serem artigos mais recentes (encontram-se nessa situação 87 artigos). Os artigos de 2009 a 2014 que não tiveram nenhuma citação foram excluídos (5 artigos). Todos os demais foram mantidos para a próxima análise que consistiu na leitura dos resumos. Então, foram eliminados 71 artigos desalinhados com o tema.

Dos 61 artigos restantes, verificou-se a disponibilidade de acesso gratuito para download, passando-se para a leitura integral (30 artigos). Da leitura dos artigos para verificação do alinhamento, três artigos foram eliminados, restando, portando, 27 estudos que compõem o banco de artigos primários.

\subsubsection{Teste de Representatividade do Portfólio Bibliográfico (PB)}

Segundo Richartz (2013), o teste de representatividade do PB consiste em analisar todas as referências bibliográficas dos 27 artigos selecionados para o banco primário, a fim de encontrar outros artigos relevantes que tenham ficado de fora da primeira busca. Encontrou-se um total de 1.179 artigos citados nas referências dos 27 artigos analisados.

Aplicou-se a mesma técnica de seleção dos primeiros artigos, isto é: artigos com publicação em periódico, no ano de 2009-2019, procedendo-se também à leitura do título para verificação de compatibilidade ao tema. Após isso, verificou-se o número de citação para o reconhecimento científico, tendo como ponto de corte os artigos que possuíssem menos de 50 citações no período estipulado acima. Contabilizando apenas uma vez aqueles artigos que apareceram em mais de uma referência, obteve-se um total de 11 artigos. 
Verificou-se que todos os 11 artigos estão disponíveis para consulta. Desses artigos, dois já fazem parte do banco de artigos primários. Para o restante, passou-se para a leitura dos resumos para verificar se estão de acordo com o tema e, posteriormente, à leitura integral. Os nove artigos estavam alinhados, portanto, somou-se um total de 27 artigos do banco primário mais nove artigos coletados das referências bibliográficas, obtendo-se, portanto, o total de 36 artigos que compõem o portfólio final.

Em destaque estão os artigos relevantes, levando-se em consideração o número de referência e o banco de artigos primários. Os 36 artigos (citados ao final na seção referências) são:

Quadro 2 - Artigos do portfólio final

\begin{tabular}{|c|c|c|}
\hline Autores & Título & LOCAL dE PUBLICAÇÃo \\
\hline $\begin{array}{l}\text { Agostino e } \\
\text { Trivieri (2014) }\end{array}$ & $\begin{array}{l}\text { Geographical Indication and wine exports. An empirical } \\
\text { investigation considering the major European producers }\end{array}$ & Food Policy \\
\hline $\begin{array}{l}\text { Albayrak e } \\
\text { Gunes (2010) }\end{array}$ & $\begin{array}{l}\text { Implementations of Geographical Indications at brand } \\
\text { management of traditional foods in the European Union }\end{array}$ & $\begin{array}{l}\text { African Journal of } \\
\text { Business Management }\end{array}$ \\
\hline $\begin{array}{l}\text { Barjolle, Paus e } \\
\text { Perret (2009) }\end{array}$ & $\begin{array}{l}\text { Impacts of Geographical Indications } \\
\text { review of methods and empirical evidences }\end{array}$ & $\begin{array}{l}\text { AgEcon - Research } \\
\text { in Agricultural \& } \\
\text { Applied Economics }\end{array}$ \\
\hline Barjolle et al. (2017) & $\begin{array}{l}\text { The role of the state for Geographical Indications of } \\
\text { coffee: case studies from Colombia and Kenya }\end{array}$ & World Development \\
\hline Belletti (2015) & $\begin{array}{l}\text { Linking protection of Geographical Indications } \\
\text { to the environment: evidence from the } \\
\text { European Union olive-oil sector }\end{array}$ & Land Use Policy \\
\hline $\begin{array}{c}\text { Belletti, } \\
\text { Marescotti e } \\
\text { Touzard (2017) }\end{array}$ & $\begin{array}{l}\text { Geographical Indications, public goods, } \\
\text { and sustainable development: the roles of } \\
\text { actors' strategies and public policies }\end{array}$ & World Development \\
\hline $\begin{array}{l}\text { Bienábi e Marie- } \\
\text { Vivien (2017) }\end{array}$ & $\begin{array}{l}\text { Institutionalizing Geographical Indications in southern } \\
\text { countries: lessons learned from Basmati and Rooibos }\end{array}$ & World Development \\
\hline $\begin{array}{l}\text { Bowen e Zapata } \\
(2009)\end{array}$ & $\begin{array}{l}\text { Geographical Indications, terroir, and socioeconomic } \\
\text { and ecological sustainability: the case of tequila }\end{array}$ & $\begin{array}{l}\text { Journal of Rural } \\
\text { Studies }\end{array}$ \\
\hline Bowen (2010a) & $\begin{array}{l}\text { Embedding local places in global spaces: Geographical } \\
\text { Indications as a territorial development strategy }\end{array}$ & Rural Sociology \\
\hline Bowen (2010b) & $\begin{array}{l}\text { Development from within? The potential } \\
\text { for Geographical Indications in the global } \\
\text { South }\end{array}$ & $\begin{array}{l}\text { The Journal of World } \\
\text { Intellectual Property }\end{array}$ \\
\hline $\begin{array}{l}\text { Dentoni, Menozzi } \\
\text { e Capelli (2012) }\end{array}$ & $\begin{array}{l}\text { Group heterogeneity and cooperation on the } \\
\text { Geographical Indication regulation: the } \\
\text { case of the "Prosciutto di Parma" consortium }\end{array}$ & Food Policy \\
\hline $\begin{array}{l}\text { Deselnicu et } \\
\text { al. (2013) }\end{array}$ & $\begin{array}{l}\text { A meta-analysis of Geographical Indication } \\
\text { food valuation studies: what drives the } \\
\text { premium for origin-based labels? }\end{array}$ & $\begin{array}{l}\text { Journal of Agricultural } \\
\text { and Resource Economics }\end{array}$ \\
\hline $\begin{array}{l}\text { Durand e Fournier } \\
\text { (2017) }\end{array}$ & $\begin{array}{l}\text { Can Geographical Indications modernize Indonesian and } \\
\text { Vietnamese agriculture? Analyzing the role of national } \\
\text { and local governments and producers' strategies }\end{array}$ & World Development \\
\hline $\begin{array}{l}\text { Galtier, Belletti e } \\
\text { Marescotti (2013) }\end{array}$ & $\begin{array}{l}\text { Factors constraining building effective and fair Geographical } \\
\text { Indications for coffee: insights from a Dominican case study }\end{array}$ & $\begin{array}{l}\text { Development } \\
\text { Policy Review }\end{array}$ \\
\hline Gangjee (2017) & $\begin{array}{c}\text { Proving provenance? Geographical Indications } \\
\text { certification and its ambiguities }\end{array}$ & World Development \\
\hline $\begin{array}{l}\text { Giovannucci, Barham } \\
\text { e Pirog (2010) }\end{array}$ & $\begin{array}{l}\text { Defining and marketing "local" foods: } \\
\text { Geographical Indications for US products }\end{array}$ & $\begin{array}{l}\text { The Journal of World } \\
\text { Intellectual Property }\end{array}$ \\
\hline
\end{tabular}




\begin{tabular}{|c|c|c|}
\hline Autores & Título & LOCAL DE PUBLICAÇÃo \\
\hline Grote (2009) & $\begin{array}{l}\text { environmental labeling, protected Geographical } \\
\text { Indications and the interests of developing countries }\end{array}$ & $\begin{array}{l}\text { The Estey Centre Journal } \\
\text { of International Law } \\
\text { and Trade Policy }\end{array}$ \\
\hline Ilbert e Petit (2009) & $\begin{array}{l}\text { Are Geographical Indications a valid property } \\
\text { right? Global trends and challenges }\end{array}$ & $\begin{array}{l}\text { Development } \\
\text { Policy Review }\end{array}$ \\
\hline Jena e Grote (2010) & $\begin{array}{l}\text { Changing institutions to protect regional heritage: a case } \\
\text { for Geographical Indications in the Indian agrifood sector }\end{array}$ & $\begin{array}{l}\text { Development } \\
\text { Policy Review }\end{array}$ \\
\hline Jena e Grote (2012) & $\begin{array}{c}\text { Impact evaluation of traditional Basmati rice cultivation } \\
\text { in Uttarakhand State of Northern India: what implications } \\
\text { does it hold for Geographical Indications? }\end{array}$ & World Development \\
\hline Kireeva (2011) & $\begin{array}{c}\text { How to register Geographical Indications } \\
\text { in the european community }\end{array}$ & World Patent Information \\
\hline $\begin{array}{l}\text { Marie-Vivien et } \\
\text { al. (2014) }\end{array}$ & $\begin{array}{c}\text { Trademarks, Geographical Indications and } \\
\text { environmental labelling to promote biodiversity: } \\
\text { the case of agroforestry coffee in India }\end{array}$ & $\begin{array}{l}\text { Development } \\
\text { Policy Review }\end{array}$ \\
\hline $\begin{array}{c}\text { Medeiros e } \\
\text { Passador (2015) }\end{array}$ & $\begin{array}{l}\text { Indicações Geográficas e turismo: } \\
\text { possibilidades no contexto brasileiro }\end{array}$ & $\begin{array}{l}\text { Perspectivas } \\
\text { contemporâneas }\end{array}$ \\
\hline $\begin{array}{l}\text { Menapace e } \\
\text { Moschini (2011) }\end{array}$ & $\begin{array}{c}\text { Quality certification by Geographical Indications, } \\
\text { trademarks and firm reputation }\end{array}$ & $\begin{array}{l}\text { Europen Review of Agricultural } \\
\text { Economics }\end{array}$ \\
\hline $\begin{array}{c}\text { Menapace e } \\
\text { Moschini (2014) }\end{array}$ & $\begin{array}{l}\text { Strength of protection for Geographical Indications: } \\
\text { promotion incentives and welfare effects }\end{array}$ & $\begin{array}{c}\text { American Journal of } \\
\text { Agricultural Economics }\end{array}$ \\
\hline $\begin{array}{l}\text { Quiñones-Ruiz } \\
\text { et al. (2016a) }\end{array}$ & $\begin{array}{l}\text { Insights into the black box of collective efforts for } \\
\text { the registration of Geographical Indications }\end{array}$ & Land Use Policy \\
\hline $\begin{array}{l}\text { Quiñones-Ruiz } \\
\text { et al. (2017) }\end{array}$ & $\begin{array}{l}\text { Why early collective action pays off: evidence from } \\
\text { setting protected Geographical Indications }\end{array}$ & $\begin{array}{l}\text { Renewable Agriculture } \\
\text { and Food Systems }\end{array}$ \\
\hline Rangnekar (2011) & $\begin{array}{c}\text { Remaking place: the social construction of } \\
\text { a Geographical Indication for Feni }\end{array}$ & $\begin{array}{l}\text { Environment } \\
\text { and Planning }\end{array}$ \\
\hline $\begin{array}{l}\text { Samaddar e } \\
\text { Samaddar (2010) }\end{array}$ & $\begin{array}{l}\text { Komal Chau - a potential candidate } \\
\text { for Geographical Indication }\end{array}$ & $\begin{array}{l}\text { Journal of Intellectual } \\
\text { Property Rights }\end{array}$ \\
\hline $\begin{array}{l}\text { Sárka e Tomás } \\
\text { (2014) }\end{array}$ & $\begin{array}{l}\text { The system of the Geographical Indication } \\
\text { - important component of the politics of the } \\
\text { consumers' protection in European Union }\end{array}$ & $\begin{array}{l}\text { Amfiteatru Economic } \\
\text { Journal }\end{array}$ \\
\hline $\begin{array}{l}\text { Sorgho e Larue } \\
\text { (2014) }\end{array}$ & $\begin{array}{l}\text { Geographical Indication regulation and } \\
\text { intra-trade in the European Union }\end{array}$ & Agricultural Economics \\
\hline Teuber (2011) & $\begin{array}{c}\text { Consumers' and producers' expectations } \\
\text { towards Geographical Indications empirical } \\
\text { evidence for a german case study }\end{array}$ & British Food Journal \\
\hline $\begin{array}{l}\text { Tregear, Torok e } \\
\text { Gorton (2016) }\end{array}$ & $\begin{array}{l}\text { Geographical Indications and upgrading of small-scale } \\
\text { producers in global agro-food chains: a case study of } \\
\text { the Mako' onion protected Designation of Origin }\end{array}$ & $\begin{array}{l}\text { Environment } \\
\text { and Planning }\end{array}$ \\
\hline Valente (2012) & $\begin{array}{c}\text { Indicação Geográfica de alimentos e bebidas } \\
\text { no Brasil e na União Europeia }\end{array}$ & Ciência Rural \\
\hline Valente et al. (2013) & $\begin{array}{l}\text { O processo de reconhecimento das Indicações Geográficas } \\
\text { de alimentos e bebidas brasileiras: regulamento de uso, } \\
\text { delimitação da área e diferenciação do produto }\end{array}$ & Ciência Rural \\
\hline $\begin{array}{l}\text { Wilkinson, Cerdan } \\
\text { e Dorigon (2017) }\end{array}$ & $\begin{array}{l}\text { Geographical Indications and "Origin" products in } \\
\text { Brazil - the interplay of institutions and networks }\end{array}$ & World Development \\
\hline
\end{tabular}

Fonte: Elaborado pelos autores deste artigo (2019) 


\subsection{Análise Bibliométrica}

Segundo Richartz (2013), na análise bibliométrica são apresentados os autores mais relevantes, os artigos e periódicos de maior destaque, assim como o reconhecimento científico dos artigos.

\subsubsection{Autores de Destaque}

Sarah K. Bowen é a autora de maior destaque. Professora de sociologia na Universidade da Carolina do Norte e Ph.D em sociologia pela Universidade de Wisconsin-Madison. Do portfólio bibliográfico desta pesquisa, isto é, dos 36 artigos selecionados, três são de Bowen. Dois de seus artigos contêm o maior número de citação dentre todos os artigos do PB (216 e 157 citações), constando da referência bibliográfica de outros seis e quatro artigos desta pesquisa, respectivamente.

Na base de dados Scopus, foram encontrados 25 documentos da autora, com uma média de 427 citações em 346 documentos durante o período de 2009-2019. Entre as áreas de interesse em pesquisa da autora estão: ciências sociais, agricultura e ciências biológicas, medicina, ciência ambiental, arte e humanidade, enfermagem, Terra e ciências planetárias, economia, econometria e finanças. Bowen tem conduzido suas pesquisas em Denominações de Origem no México, na França e nos Estados Unidos.

Na sequência, destacam-se os autores: Giovanni Belletti e Andrea Marescotti, ambos do Dipartimento di Scienze per l'Economia e l'Imprensa da Universidade de Florence, e Jean-Marc Touzard, do French National Institute for Agricultural Research.

\subsubsection{Periódicos Relevantes}

Os periódicos utilizados pelos artigos do portfólio foram analisados e constatou-se que o mais relevante é o World Development com sete artigos do PB publicados. Trata-se de uma revista mensal multidisciplinar de estudos de desenvolvimento que mantém o foco na cooperação global para melhorar os padrões de vida e a condição humana em geral.

Em seguida, destacam-se os periódicos Development Policy Review -4, The Jornal of World Intellectual Property - 3 e Journal of Agricultural and Resource Economics - 3. 
Figura 1 - Periódicos mais relevantes

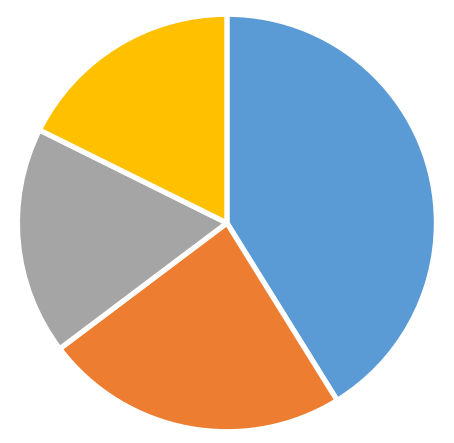

- World Development

- Development Policy Review

- The jornal of World Intellectual Property

- Journal of agricultural and Resource Economics

Fonte: Elaborada pelos autores deste artigo (2019)

\subsubsection{Artigos de Destaque}

De acordo com o número de citações no Google Scholar, os artigos do portfólio destacados nesta pesquisa são: Geographical Indications, terroir, and socioeconomic and ecological sustainability: the case of tequila (2009), de Bowen e Zapata. Esse artigo teve o maior número de citações (216) e foi citado por outros seis artigos do PB. O artigo analisa o potencial social, econômico e os impactos ecológicos das Indicações Geográficas utilizando como estudo o caso da tequila.

A seguir, com 157 citações, destaca-se o artigo Embedding local places in global spaces: Geographical Indications as a territorial development strategy (2010), da já citada autora de destaque Sarah k. Bowen. Ela compara dois sistemas de produção da IG e destaca a atuação do Estado para nivelar o campo de atuação e a capacitação de pequenos agricultores como pré-condição para o sucesso da Indicação Geográfica. Esses artigos foram encontrados nas referências bibliográficas. O terceiro artigo destacado, por outro lado, já fazia parte do banco de artigos primários: Geographical Indications, public goods, and sustainable development: the roles of actors'strategies and public policies (2017), tem como autores Giovanni Belletti, Andrea Marescotti e Jean-Marc Touzard e foi citado 70 vezes.

\subsection{Análise Sistêmica}

A análise sistêmica é a interpretação de artigos com base em critérios predefinidos e foi realizada com base nos critérios constantes no Quadro 1, permitindo um aprofundamento do tema das Indicações Geográficas.

\subsubsection{Critério 1 - Natureza}

Com relação à natureza dos artigos do PB (teóricos ou teóricos-empíricos), observou-se que, dos 36 artigos que fazem parte do portfólio bibliográfico, seis são considerados estudos 
teóricos e 30 são classificados como teóricos-empíricos, isto é, pressupõe a comprovação prática por meio de diversos métodos aplicados a um contexto específico.

\subsubsection{Critério 2 - Objeto}

O objeto tem por finalidade identificar onde se concentram as pesquisas. Identificou-se que as pesquisas se concentram nos seguintes países: México, França, Alemanha, Estados Unidos, União Europeia, Índia, Brasil, Indonésia, Itália, Áustria, República Dominicana, Turquia, Hungria, Colômbia, África e China.

Grande parte dos estudos tem como referência países da União Europeia devido ao histórico e influência que as Indicações Geográficas possuem nesses países. Outros artigos concentram suas pesquisas nos países em desenvolvimento diante do enorme potencial de aplicação da IG nessas regiões.

\subsubsection{Critério 3 - Técnicas}

Essa lente busca identificar a metodologia utilizada em cada um dos artigos mapeados. O resultado demonstra o predomínio de artigos qualitativos, que utilizam técnicas de pesquisa de campo por meio de entrevistas com atores locais, questionários, bem como pesquisa bibliográfica e documental. A entrevista parece ser a técnica mais utilizada na investigação da realidade de cada região.

\subsubsection{Critério 4 - Vantagens}

Por meio dessa lente, buscou-se identificar nos artigos mapeados as vantagens da Indicação Geográfica sobre diferentes cenários e produtos. O resultado está estruturado no Quadro 3.

Quadro 3 - Vantagens da obtenção de Indicação Geográfica

\begin{tabular}{|c|c|}
\hline VANTAGENS DA OBTENÇÃo DE INDICAÇÃo GEOGRÁFICA & AutORES \\
\hline Proteção contra imitações & $\begin{array}{l}\text { Belletti et al. (2009), Giovannucci } \\
\text { et al. (2009), Teuber (2011) }\end{array}$ \\
\hline Prêmios de preços & $\begin{array}{l}\text { Belletti et al. (2009), Giovannucci et } \\
\text { al. (2009), Grote (2009), Deselnicu et } \\
\text { al. (2013), Jena e Grote (2012) }\end{array}$ \\
\hline Acesso a novos canais de marketing & $\begin{array}{l}\text { Belletti et al. (2009), Giovannucci et al. (2009), } \\
\text { Teuber (2011), Jena e Grote (2012) }\end{array}$ \\
\hline Aumento e desenvolvimento do turismo & Valente et al. (2012), Medeiros e Passador (2015) \\
\hline Desenvolvimento rural & Belletti et al. (2015a), Bowen e Zapata (2009) \\
\hline Geração de empregos e aumento da renda & Belletti et al. (2015a), Bowen (2010) \\
\hline Manutenção de formas tradicionais de produção & Bowen (2010) \\
\hline Liderança forte & Bowen (2010) \\
\hline Visão coletiva orientada para sustentabilidade e qualidade & Bowen (2010), Bowen e Zapata (2009) \\
\hline Organização justa e representativa dos agentes locais & Bowen (2010) \\
\hline
\end{tabular}




\begin{tabular}{|c|c|}
\hline VANTAGENS DA OBTENÇÃo DE INDICAÇÃo GEOGRÁFICA & AutORES \\
\hline Modernização e melhoramento dos meios de produção & Bowen (2010) \\
\hline Esforços coletivos & Galtier, Belletti e Marescotti (2013) \\
\hline Proteção do patrimônio cultural & Teuber (2011) \\
\hline Garantia ao consumidor da qualidade do produto & Teuber (2011) \\
\hline Ligação com o terroir & Bowen e Zapata (2009) \\
\hline Desenvolvimento rural & Giovannucci, Barham e Pirog (2010); Grote (2009) \\
\hline Abrir novos mercados de exportação & Grote (2009) \\
\hline Promover da imagem do produto & Grote (2009) \\
\hline Informação sobre produtos & Jena e Grote (2012) \\
\hline Benefício aos consumidores & Gangjee (2017) \\
\hline Maior interação entre produtores gera melhores resultados & Quiñones-Ruiz et al. (2016) \\
\hline
\end{tabular}

Fonte: Dados da pesquisa (2019)

\subsubsection{Critério 5 - Desafios}

Com relação aos desafios enfrentados na obtenção da Indicação Geográfica, o mapeamento possibilitou identificar os seguintes resultados, apresentado no Quadro 4.

Quadro 4 - Desafios da obtenção de Indicação Geográfica

\begin{tabular}{|c|c|}
\hline DESAFIOS DA OBTENÇão DE INDICAÇão GEOGRÁFICA & AutoRES \\
\hline Ausência de estrutura institucional forte & Teuber (2011), Gangjee (2017) \\
\hline Normas regulamentareis excessivamente abertas & Bowen (2010) \\
\hline Competição com grandes indústrias & Bowen (2010) \\
\hline Descaracterização dos meios tradicionais de produção & Bowen (2010), Bowen e Zapata (2009) \\
\hline Degradação ambiental & Zapata (2005), Bowen; Zapata (2009). \\
\hline Perda da biodiversidade & Zapata (2005), Bowen; Zapata (2009). \\
\hline Ausência de participação do Estado & Bowen (2010) \\
\hline Grande concorrência & Bowen (2010) \\
\hline Falta de ligação do terroir & Bowen e Zapata (2009) \\
\hline Baixa qualidade do produto & Bowen e Zapata (2009) \\
\hline Ineficácia para realizar o desenvolvimento rural & Bowen e Zapata (2009) \\
\hline Falta de recurso do pequeno produtor, \\
dificultando na aquisição da IG \\
\hline $\begin{array}{c}\text { Dificuldade de transmitir informações } \\
\text { sobre a IG aos consumidores }\end{array}$ & $\begin{array}{c}\text { Giovannucci, Barham e Pirog } \\
(2010), \text { Grote (2009) }\end{array}$ \\
\hline Parâmetros de proteção pouco transparentes & Giovannucci, Barham e Pirog (2010) \\
\hline $\begin{array}{c}\text { Pouco conhecimento da população local e de iniciativas } \\
\text { públicas gerando um processo de IG demorado }\end{array}$ & Biénabe e Marie-Vivien (2015) \\
\hline
\end{tabular}




\begin{tabular}{|c|c|}
\hline DeSAFIOS DA OBTENÇão DE INDICAÇão GEOGRÁFICA & AutorES \\
\hline $\begin{array}{c}\text { Falta de agentes locais com capacidades de } \\
\text { controlar qualidade e origem do produto } \\
\text { Ausência de participação dos produtores }\end{array}$ & Biénabe e Marie-Vivien (2015) \\
\hline Falta de parceiros de mercado fortes & Gangjee (2017) \\
\hline Proteção legal efetiva & Gangjee (2017) \\
\hline $\begin{array}{c}\text { Falta de acesso, educação e treinamento } \\
\text { que tragam valor ao produto }\end{array}$ & Gangjee (2017) \\
\hline Ausência de competição no mercado local & Gám (2014) \\
\hline
\end{tabular}

Fonte: Dados da pesquisa (2019)

\subsubsection{Critério 6 - Aplicabilidade}

No quesito aplicabilidade, a análise demonstra que a maioria dos artigos mapeados traz cases de diferentes localidades e contextos que utilizaram a proteção da Indicação Geográfica. Desse modo, por meio desse panorama, foram obtidos resultados que expõem as melhores maneiras de aplicação da IG, além de identificar os pontos de melhoramento para uma melhor experiência com a proteção.

\section{Considerações Finais}

O objetivo deste artigo foi realizar um mapeamento das pesquisas científicas acerca do tema da Indicação Geográfica, a fim de identificar seu panorama atual $e$ as possibilidades de auferir vantagens e resolver os desafios de sua aplicação. Para tanto, efetuou-se pesquisa em bases de dados, seguindo critérios específicos. Para selecionar um portfólio bibliográfico (PB) relevante, realizou-se, então, a análise bibliométrica desses artigos, bem como de suas referências; $e$, por fim, efetuou-se a análise sistêmica (análise de conteúdo) com os artigos do PB sob a ótica da Indicação Geográfica.

O processo de seleção do banco de artigos resultou em 36 artigos que compõem o Portfólio Bibliográfico, de acordo com os critérios definidos na pesquisa. O destaque refere-se ao artigo de Sarah K. Bowen (2010). O principal periódico é o World Development com sete artigos do PB publicados.

Em relação à análise sistêmica, critério 1, pode-se concluir que, dos 36 artigos que fazem parte do portfólio bibliográfico, seis são considerados estudos teóricos e 30 são classificados como teóricos-empíricos. No que se refere ao quesito objeto, critério 2 , percebe-se que as pesquisas se concentram nos seguintes países: México, França, Alemanha, Estados Unidos, União Europeia, Índia, Brasil, Indonésia, Itália, Áustria, República Dominicana, Turquia, Hungria, Colômbia, África e China. O resultado do critério 3 demonstra o predomínio de artigos qualitativos, com a utilização da técnica de entrevistas com os agentes locais.

Observa-se que, apesar da importância do tema, ele é mais difundido em países que já possuem uma longa tradição na proteção da IG. Nos países em desenvolvimento, como o Brasil, o IG precisa ser mais explorado. Para os critérios 4 e 5 são identificados alguns desafios e 
vantagens expostos no Quadro 3 e Quadro 4. Entre as principais vantagens identificadas nos artigos mapeados, além da proteção contra imitação, é possível citar o desenvolvimento rural e do turismo da região e a valorização do produto. Quanto aos principais desafios verificados, destaca-se a dificuldade de correlacionar o produto ao território $e$ a falta de informação $e$ conhecimento sobre a Indicação Geográfica. Por fim, com relação à aplicabilidade, a maioria dos artigos mapeados traz cases de diferentes localidades e contextos que utilizaram a proteção da Indicação Geográfica e, por meio desse panorama, foram obtidos resultados expondo as melhores maneiras de aplicação da IG ou identificando os pontos de melhoramento para uma melhor experiência com a proteção.

Ressalta-se que as conclusões deste estudo se limitam à visão do pesquisador, por meio da construção de critérios e lentes específicas, conforme apontam os objetivos traçados. Portanto, as conclusões dizem respeito ao portfólio bibliográfico definido pelos critérios deste artigo.

\section{Referências}

AFONSO, M. H. F. et al. Como construir conhecimento sobre o tema de pesquisa? Aplicação do processo Proknow- $\mathrm{C}$ na busca de literatura sobre avaliação do desenvolvimento sustentável. Revista de Gestão Social e Ambiental, [s.l.], v. 5, n. 2, p. 47-62, 2011.

AGOSTINO, Mariarosaria; TRIVIERI, Francesco. Geographical indication and wine exports. An empirical investigation considering the major European producers. Food Policy, [s.l.], v. 46, p. 2236, 2014.

ALBAYRAK, Mevhibe; GUNES, Erdoğan. Implementations of geographical indications at brand management of traditional foods in the European Union. African Journal of Business Management, [s.l.], v. 4, n. 6, p. 1.059-1.068, 2010.

AZEVEDO, R. C. et al. Avaliação de desempenho do processo de orçamento: estudo de caso em uma obra de construção civil. Ambiente Construído (Online), [s.l.], v. 11, p. 85-104, jan.-mar. 2011.

BARJOLLE, Dominique; PAUS, Marguerite; PERRET, Anna O. Impacts of geographical indications-review of methods and empirical evidences. 2009.

BARJOLLE, Dominique et al. The Role of the State for Geographical Indications of Coffee: Case Studies from Colombia and Kenya. World Development, [s.l.], v. 98, p. 105-119, 2017.

BELLETTI, Giovanni et al. Linking protection of geographical indications to the environment: Evidence from the European Union olive-oil sector. Land Use Policy, [s.l.], v. 48, p. 94-106, 2015.

BELLETTI, Giovanni; MARESCOTTI, Andrea; TOUZARD, Jean-Marc. Geographical indications, public goods, and sustainable development: The roles of actors' strategies and public policies. World Development, [s.l.], v. 98, p. 45-57, 2017.

BIÉNABE, Estelle; MARIE-VIVIEN, Delphine. Institutionalizing geographical indications in southern countries: lessons learned from Basmati and Rooibos. World Development, [s.l.], v. 98, p. 58-67, 2017.

BOWEN, Sarah; ZAPATA, Ana Valenzuela. Geographical indications, terroir, and socioeconomic and ecological sustainability: The case of tequila. Journal of Rural Studies, [s.l.], v. 25, n. 1, p. 108119, 2009. 
BOWEN, Sarah. Embedding local places in global spaces: Geographical indications as a territorial development strategy. Rural Sociology, [s.l.], v. 75, n. 2, p. 209-243, 2010.

BOWEN, Sarah. Development from within? The potential for geographical indications in the global south. The Journal of World Intellectual Property, [s.l.], v. 13, n. 2, p. 231-252, 2010.

BORTOLUZZI, S. C. et al. Avaliação de desempenho em redes de pequenas e médias empresas: estado da arte para as delimitações postas pelo pesquisador. Revista Eletrônica Estratégia \& Negócios, [s.l.], v. 4, n. 2, 2011.

BRASIL. Lei n. 9.279, de 14 de maio de 1996. Regula sobre direitos e obrigações relativos à propriedade intelectual. Disponível em: http://www.planalto.gov.br/ccivil_03/leis/19279.htm. Acesso em: 3 mar. 2021.

CHAVES, L. C. Sistemas de apoio à decisão: mapeamento e análise de conteúdo. Revista Eletrônica de Ciência Administrativa. [s.l.], v. 12, n. 1, p. 6-22. 2013.

DESELNICU, Oana C. et al. A meta-analysis of geographical indication food valuation studies: what drives the premium for origin-based labels? Journal of Agricultural and Resource Economics, [s.l.], p. 204-219, 2013.

DENTONI, Domenico; MENOZZI, Davide; CAPELLI, Maria Giacinta. Group heterogeneity and cooperation on the geographical indication regulation: The case of the "Prosciutto di Parma" Consortium. Food Policy, [s.l.], v. 37, n. 3, p. 207-216, 2012.

DE LIMA MEDEIROS, Mirna; PASSADOR, João Luiz. Indicações Geográficas e Turismo: possibilidades no contexto brasileiro. Perspectivas Contemporâneas, [s.l.], v. 10, n. 3, p. 56-79, 2015.

DURAND, Claire; FOURNIER, Stéphane. Can geographical indications modernize Indonesian and Vietnamese agriculture? Analyzing the role of national and local governments and producers' strategies. World Development, [s.l.], v. 98, p. 93-104, 2017.

ENSSLIN, L.; ENSSLIN, S. R.; PACHECO, G. C. Um estudo sobre segurança em estádios de futebol baseado na análise bibliométrica da literatura internacional. Perspectivas em Ciência da Informação, [s.l.], v. 17, n. 2, p. 71-91, 2012.

ENSSLIN, L. et al. Avaliação do desempenho de empresas terceirizadas com o uso da metodologia multicritério de apoio à decisão -construtivista. Pesquisa Operacional, [s.l.], v. 30, p. 125-152, 2010.

ENSSLIN, L.; ENSSLIN, S. R.; PINTO, H. M. Processo de investigação e análise bibliométrico: avaliação da qualidade dos serviços bancários. Revista de Administração Contemporânea, [s.l.], v. 17, n. 3, p. 325-349, 2013.

FLORES, S. S.; TONIETTO, J.; TAFFAREL, J. C. Painel de indicadores para avaliação das indicações geográficas de vinhos brasileiros. Cadernos de Prospecção, Salvador, v. 12, n. 4, 2019.

GALTIER, Franck; BELLETTI, Giovanni; MARESCOTTI, Andrea. Factors constraining building effective and fair geographical indications for coffee: Insights from a Dominican case study. Development Policy Review, [s.l.], v. 31, n. 5, p. 597-615, 2013.

GANGJEE, Dev S. Proving provenance? Geographical indications certification and its ambiguities. World Development, [s.l.], v. 98, p. 12-24, 2017. 
GIOVANNUCCI, Daniele; BARHAM, Elizabeth; PIROG, Richard. The Journal of World Intellectual Property, [s.l.], v. 13, n. 2, p. 94-120, 2010.

GROTE, Ulrike. Environmental labeling, protected geographical indications and the interests of developing countries. Estey Journal of International Law and Trade Policy, [s.l.], v. 10, n. 17532016-141181, p. 94, 2009.

ILBERT, Hélène; PETIT, Michel. Are geographical indications a valid property right? Global trends and challenges. Development Policy Review, [s.l.], v. 27, n. 5, p. 503-528, 2009.

INPI - INSTITUTO NACIONAL DA PROPRIEDADE INDUSTRIAL. Instrução Normativa n. 95, de 28 de dezembro de 2018. [2018]. Disponível em: file://C:/Users/drimaues/Downloads/ IN952018\%20(2).pdf. Acesso em: 28 nov. 2019.

JENA, Pradyot R.; GROTE, Ulrike. Changing institutions to protect regional heritage: a case for geographical indications in the Indian agrifood sector. Development Policy Review, [s.l.], v. 28, n. 2, p. 217-236, 2010.

JENA, Pradyot R.; GROTE, Ulrike. Impact evaluation of traditional Basmati rice cultivation in Uttarakhand State of Northern India: what implications does it hold for Geographical Indications? World Development, [s.l.], v. 40, n. 9, p. 1.895-1.907, 2012.

KIREEVA, Irina. How to register geographical indications in the European Community. World Patent Information, [s.l.], v. 33, n. 1, p. 72-77, 2011.

LACERDA, R. T. O.; ENSSLIN, L.; ENSSLIN, S. R. A performance measurement framework in portfolio management: A constructivist case. Management Decision, [s.l.], v. 49, n. 3- 4, p: 648668, 2011.

LACERDA, R. T. O.; ENSSLIN, L.; ENSSLIN, S. R. Uma análise bibliométrica da literatura sobre estratégia e avaliação de desempenho. Gestão \& Produção, [s.l.], v. 19, p. 59-78, 2012.

MARIE-VIVIEN, Delphine et al. Trademarks, geographical indications and environmental labelling to promote biodiversity: the case of agroforestry coffee in India. Development Policy Review, [s.l.], v. 32, n. 4, p. 379-398, 2014.

MAPA - MINISTÉRIO DA AGRICULTURA, PECUÁRIA E ABASTECIMENTO. [2019]. Disponível em: http://www.agricultura.gov.br/. Acesso em: 19 abr. 2019.

MARINS, M. F.; CABRAL, D. H. Q. O papel da indicação geográfica como propulsor da inovação e do desenvolvimento local: caso vale dos vinhedos. Cadernos de Prospecção, Salvador, v. 8, n. 2, 2015.

MENAPACE, Luisa; MOSCHINI, Gian Carlo. Quality certification by geographical indications, trademarks and firm reputation. European Review of Agricultural Economics, [s.l.], v. 39, n. 4, p. 539-566, 2011.

MENAPACE, Luisa; MOSCHINI, Gian Carlo. Strength of protection for geographical indications: promotion incentives and welfare effects. American Journal of Agricultural Economics, [s.l.], v. 96, n. 4, p. 1.030-1.048, 2014.

QUINONES-RUIZ, Xiomara F. et al. Insights into the black box of collective efforts for the registration of geographical indications. Land Use Policy, [s.l.], v. 57, p. 103-116, 2016. 
QUIÑONES-RUIZ, Xiomara F. et al. Why early collective action pays off: evidence from setting Protected Geographical Indications. Renewable Agriculture and Food Systems, [s.l.], v. 32, n. 2, p. 179-192, 2017.

RANGNEKAR, Dwijen. Remaking place: The social construction of a geographical indication for Feni. Environment and Planning A, [s.l.], v. 43, n. 9, p. 2.043-2.059, 2011.

RICHARTZ, Fernando; ENSSLIN, Sandra Rolim. Comportamento dos custos: mapeamento e análise sistêmica das publicações internacionais. In: CONGRESSO BRASILEIRO DE CUSTOS-ABC. 2013. Anais [...]. 2013.

RICHARDSON, R. J. Pesquisa social: métodos e técnicas. 3. ed. São Paulo: Atlas, 1999.

ROSA, F. S.; ENSSLIN, S. R.; ENSSLIN, L. Evidenciação ambiental: processo estruturado de revisão de literatura sobre avaliação de desempenho da evidenciação ambiental. Sociedade, Contabilidade e Gestão, [s.l.], v. 4, n. 2, p. 24-37, 2009.

RODRIGUES VALENTE, Maria Emília et al. Indicação geográfica de alimentos e bebidas no Brasil e na União Europeia. Ciência Rural, [s.l.], v. 42, n. 3, 2012.

SAMADDAR, S. G.; SARNADDAR, A. B. Komal Chaul - a potential candidate for Geographical Indication. Journal of Intelectual Property Right, [s.l.], 2010.

SORGHO, Zakaria; LARUE, Bruno. Geographical indication regulation and intra-trade in the European Union. Agricultural Economics, [s.l.], v. 45, n. S1, p. 1-12, 2014.

TASCA, J. E. et al. An approach for selecting a theoretical framework for the evaluation of training programs. Journal of European Industrial Training, [s.l.], v. 34, n. 7, p. 631-655. 2010.

TEUBER, Ramona. Consumers' and producers' expectations towards geographical indications: Empirical evidence for a German case study. British Food Journal, [s.l.], v. 113, n. 7, p. 900-918, 2011.

TREGEAR, Angela; TÖRÖK, Áron; GORTON, Matthew. Geographical indications and upgrading of small-scale producers in global agro-food chains: A case study of the Makó Onion Protected Designation of Origin. Environment and Planning A, [s.l.], v. 48, n. 2, p. 433-451, 2016.

WILKINSON, John; CERDAN, Claire; DORIGON, Clovis. Geographical Indications and "Origin" Products in Brazil-The Interplay of Institutions and Networks. World Development, [s.l.], v. 98, p. 82-92, 2017.

VALMORBIDA, S. M. I. et al. Gestão pública com foco em resultados: evidenciação de oportunidades de pesquisa. Revista CAP - Accounting and Management, [s.l.], v. 5, n. 5, 2011.

VALENTE, Maria Emília Rodrigues et al. O processo de reconhecimento das indicações geográficas de alimentos e bebidas brasileiras: regulamento de uso, delimitação da área e diferenciação do produto. Ciência Rural, [s.l.], v. 43, n. 7, p. 1.330-1.336, 2013.

VELCOVSKÁ, Šárka; SADÍLEK, Tomáš. The System of the Geographical Indication - Important Component of the Politics of the Consumers' Protection in European Union. Amfiteatru Economic Journal, [s.l.], v. 16, n. 35, p. 228-242, 2014.

VIANNA, W. B.; ENSSLIN, L.; GIFFHORN, E. A integração sistêmica entre pós-graduação e educação básica no Brasil: contribuição teórica para um "estado da arte". Ensaio: Avaliação e Políticas Públicas em Educação, [s.l.], v. 19, n. 71, p. 327-344. 2011. 


\section{Sobre os Autores}

\section{Adriana Araújo Maués}

E-mail: adrianamaues@gmail.com

Mestre em propriedade intelectual e transferência de tecnologia para inovação pela Universidade Federal de Santa Catarina em 2020.

Endereço profissional: PROFNIT, Departamento de Ciências Contábeis, Bloco C, Rua Desembargador Vitor Lima, n. 222, Trindade, Florianópolis, SC. CEP: 88040-400.

\section{Fernando Richartz}

E-mail: fernandorichartz@gmail.com

Doutor em contabilidade pela Universidade Federal de Santa Catarina em 2016.

Endereço profissional: Centro Socioeconômico, Departamento de Ciências Contábeis, sala 114, Campus Universitário Reitor João David Ferreira Lima, s/n, Trindade, Florianópolis, SC. CEP: 88040-900. 